\section{OCCURRENCE OF ARTHROPATIES IN CHILDREN WITH INFLAMMATORY BOWEL DISEASES}

\section{E. Szakos ${ }^{1}$, E. Borbas ${ }^{2}$, I. Huszanyik ${ }^{3}$, A. Monus ${ }^{1}$, E. Solyom ${ }^{1}$, K. Tamasi ${ }^{1}$ \\ 13rd Department of Pediatrics, Pediatric Health Centre, ${ }^{2}$ Department of Pediatric Radiology, Pediatric Health Centre, ${ }^{3}$ Department of Orthopedic Surgery, Borsod-Abauj-Zemplen County University Hospital, Miskolc, Hungary}

Objectives and study: Arthritis associated with inflammatory bowel diseases is mainly part of systemic sterile inflammation.

Authors studied occurrence of locomotive system's inflammation, by prospective and partly retrospective methods in 35 pediatric cases (17 boys and 18 girls) with inflammatory bowel disease (IBD), carried out in Pediatric Health Centre, Borsod-Abauj-County University Hospital, Miskolc, Hungary.

Methods: Data of patients history, physical (pediatric gastroenterologist, orthopedic surgeon), X-ray, and/ or ultrasound examinations were analized.

Results: Diagnosis of IBD was performed at 4.517 years of age. Signs of locomotive system's inflammation appeared before, in same time, or after diagnosing bowel's inflammation. 9/35 (25.7\%) patients presented knee-joint gout, swelling, synovial fluid production. $18 / 35(50.3 \%)$ of them had migrating peripherial arthitis, $4 / 35(11.4 \%)$ axial form. Transient rheumatic factor positivity appeared in $2 / 35$ patients (5.5\%). 29/35 pediatric IBD patients have some joint-mouvement system's complaints. Drugs of IBD therapy (nonsteroid antiinflammatory drugs, corticosteroids, azathioprin, biologics) were effective in associated arthritis also.

Conclusion: Antiinflammatory-immunesupressive drugs with systemic effect ease inflammation not only in bowel, but in joints also. It is important to consider joint inflammation when doctors propose therapy of patients with IBD.

\section{PROGRESSIVE FAMILIAL INTRANHEPATIC CHOLESTASIS WITH LOW \\ GAMMAGLUTAMYLTRANSFRASIS: TUNISIAN EXPERIENCE ABOUT 45 CASES}

\author{
E. Barkaoui ${ }^{1}$, A. Ayadi ${ }^{2}$, M. Chaabouni ${ }^{3}$,
} M. Hachicha ${ }^{4}$, A. Maherzi ${ }^{5}$, S. Abbès ${ }^{1}$

${ }^{1}$ Pasteur Institut, Tunis, ${ }^{2}$ Children Hospital, Mahdia, ${ }^{3}$ Hedi Chaker Hospital, ${ }^{4}$ Children Center, Sfax, ${ }^{5}$ Children Center, Marsa, Tunisia

Background and aims: PFIC has been distinguished from other forms of cholestatic liver disease in childhood by clinical, biological and morphologic studies. Molecular biology permet to confirm the diagnosis and to distinguish tow forms ( PFIC1 and 2) due to tow separate genes: ATP8B1 and ABCB11.

The Aim of our study is to investigate the clinical aspects of PFIC with low GGT in Tunisian patients and to establish standard strategy of management.

Patients and methods: Forty five patients from 22 unrelated families were retrospectively reviewed (1995-2009) from 3 paediatric centers. The diagnosis was performed by combination of clinical (pruritus), biological (cholestasis with normal or low level of GGT and normal level of cholesterol), histological features, familial history and high level of serum primary bile acids.

Results: The age at presentation ranged from the neonatal period to 12 months. Consanguinity was found in $85 \%$. Pruritus and hepatomegalia are constant. Fourteen infants had lichenification and enlargement of hands and feet. For infants have vesicular lithiasis $(12.5 \%)$. Response to AUCD treatment was in 55\%. Eleven infants (34\%) developed portal features. Nine infants (28\%) were dead at age from 5 months to 15 years old. Tow cases have an intense pruritis managed with high dose of rifampicin and sertraline. For cases had extrahepatic futures (pancreatitis and Beer distruption).

Conclusion: The prognosis of the infants with PFIC will be ameliorated by the developement of hepatic transplantation. Molecular biology is a sure tool of diagnosis and antenatal diagnosis will be soon performed in Tunisia. 